\title{
A CLASS OF FUNCTIONAL EQUATIONS AND MIELNIK PROBABILITY SPACES
}

\author{
S. J. GUCCIONE, JR. AND Č. V. STANOJEVIĆ
}

\begin{abstract}
Let $S$ be the unit sphere of a normed real linear space $N$ and let $(S, p)$ be a Mielnik space of dimension two. For $p(x, y)=f(\|x+y\|), x, y$ $\in S$, where $f$ is a continuous, strictly increasing function from $[0,2]$ onto $[0$, 1], it has been shown that $(S, p)$ being two dimensional is equivalent to $N$ being an inner product space.

In some polarization problems modeled on the unit sphere of an inner product space, the transition probability $p(x, y)$ may not be as well behaved as $p(x, y)=f(\|x+y\|)$. In order to provide a more suitable setting, we have constructed wide classes of two-dimensional transitional probability spaces $(S, p)$, all having the same set of bases $\mathscr{B}$, with $p=\phi \circ f$ where $\phi$ is a solution of a certain functional equation. In particular, for $p(x, y)=\|x+y\|^{2} / 4$, we answer a question due to B. Mielnik.
\end{abstract}

1. Introduction. In [1], transitional probability spaces, in the sense of Mielnik [2], were utilized to obtain a new characterization of real inner product spaces.

Let $\mathscr{F}=\{f \mid f:[0,2]$ onto $[0,1], f$, continuous and strictly increasing $\}$ and let $\mathcal{G}=\{g \mid g:[0,2]$ onto $[0,2], g$, continuous and strictly decreasing $\}$. Considering those $f \in \mathscr{F}$ and $g \in \mathcal{G}$ that satisfy the functional equation $f+f \circ g=1$ where $(f \circ g)(t)=f(g(t))$ and 1 is the identity function on [0,2], the following result is given in [1]: Let $N$ be a normed real linear space, $S$ its unit sphere, and let $p(x, y)=f(\|x+y\|)$, where $f \in \mathscr{F}$. Then $N$ is an inner product space if and only if, for some $f \in \mathscr{F},(S, p)$ is a Mielnik probability space of dimension 2.

This result provides an adequate model for some polarization problems. However, there are polarizations of particles other than photons modeled on the unit sphere $S$ of a real inner product space $N$ for which transitional probabilities $p(x, y)$ of the form $p(x, y)=f(\|x+y\|)$ are not adequate.

The above remark motivates an effort to study two-dimensional transitional probability spaces that will provide a more suitable setting for this kind of polarization phenomena.

In order to construct those more suitable transitional probability spaces, we need to consider functions satisfying the following functional equation:

$$
\phi(t)+\phi(1-t)=1, \quad t \in[0,1] .
$$

Presented to the Society, January 23, 1976; received by the editors December 16, 1975.

AMS (MOS) subject classifications (1970). Primary 46C10; Secondary 81A12.

Key words and phrases. Mielnik probability spaces, functional equation. 
The size of the class $\Phi_{0}$ of all functions $\phi$ from $[0,1]$ onto $[0,1]$ and satisfying $(*)$ is too wide for our purposes, as the following proposition indicates.

Proposition 1. Every $\phi \in \Phi_{0}$ is of the form

$$
\phi(t)=\psi\left(t-\frac{1}{2}\right)+\frac{1}{2}, \quad t \in[0,1]
$$

where $\psi$ is an odd function on $\left[-\frac{1}{2}, \frac{1}{2}\right]$.

Any of the following subclasses of $\Phi_{0}$ can be used in the construction of more general transitional probabilities:

$\Phi_{1}=\left\{\phi \mid \phi \in \Phi_{0}, \phi(t)=0 \Leftrightarrow t=0\right\}$,

$\Phi_{2}=\left\{\phi \mid \phi \in \Phi_{0}, \phi\right.$, strictly increasing $\}$,

$\Phi_{3}=\left\{\phi \mid \phi \in \Phi_{0}, \phi\right.$, strictly increasing and continuous $\}$.

It is plain that $\Phi_{3} \subset \Phi_{2} \subset \Phi_{1} \subset \Phi_{0}$. Even the smallest subclass $\Phi_{3}$ can exhibit very pathological behavior as is shown by Ganguli [3]; for instance, $\Phi_{3}$ contains Cantor's singular function.

In general, polarization does not have to be a mapping $T: x \mapsto-x$ where $x$ is a unit vector in $N$. It can be modeled by a corresponding Mielnik twodimensional probability space with an automorphism $T: S \rightarrow S$ where $S$ is any set of states.

The following proposition makes the above remark even more transparent.

Proposition 2 [1]. Let $(S, p)$ be a two-dimensional Mielnik space. Then there is an involution $T: S \rightarrow S$ such that every basis $B$ of $(S, p)$ is of the form $B=\{x, T x\}, x \in S$.

In particular, if $S$ is the unit sphere of a normed real linear space $N$, then $T x=-x$ as shown in [1].

2. Construction of two-dimensional transitional probability spaces. We are now in the position to exhibit the existence of a wide class of two-dimensional probability spaces in a very general setting utilizing the class $\Phi_{1}$.

TheOREM. Let $(S, p)$ be a Mielnik probability space of dimension 2. Then for every $\phi \in \Phi_{1},(S, \phi \circ p)$ is a transitional probability space of dimension 2 , where $\phi \circ p=\phi(p)$.

Proof. Since $\phi$ is a self-map of $[0,1]$ and $0 \leqslant p(x, y) \leqslant 1,0 \leqslant \phi(p(x, y))$ $\leqslant 1$ for all $x, y \in S$.

If $\phi(p(x, y))=1$, then from $(*)$ we obtain $\phi(1-p(x, y))=0$. Since $\phi(t)$ $=0$ if and only if $t=0$, this implies that $p(x, y)=1$. Since $p(x, y)$ is a probability function, this forces $x=y$.

If $x=y$, then $p(x, x)=1$ and, hence, $\phi(p(x, x))=\phi(1)=1$ by definition of the class $\Phi_{1}$. Therefore, $\phi(p(x, y))=1$ if and only if $x=y$. The symmetry of $\phi(p(x, y))$ follows from the symmetry of $p(x, y)$. Now, $\phi(p(x, T x))=\phi(0)$ 
$=0$, since $(S, p)$ is two dimensional with all bases of the form $B=\{x, T x\}, x$ $\in S$, and $\phi(0)=0$. Moreover, if $\phi(p(x, y))=0$, then $p(x, y)=0$. Since $(S, p)$ is a two-dimensional Mielnik space, this forces $y=T x$.

Therefore, all bases $\mathscr{B}$ of $(S, \phi \circ p)$ are of the form $B=\{x, T x\}, x \in S$. Since $\phi$ is a solution of $(*)$ and $p(x, y)$ satisfies Axiom $C$ in [2], we have, for any $x \in S$ and basis $B=\{y, T y\}, y \in S$,

$$
\phi(p(x, y))+\phi(p(x, T y))=\phi(p(x, y))+\phi(1-p(x, y))=1,
$$

i.e., $\phi \circ p$ satisfies Axiom C. Hence, $(S, \phi \circ p)$ is a two-dimensional transitional probability space.

By taking $S$ to be the unit sphere of a normed real linear space and $p(x, y)=f(\|x+y\|)$ to be any function of $\|x+y\|$ satisfying the generalization of the parallelogram law given in [1], we obtain the following corollary.

COROLlaRy 1. Let $N$ be a real inner product space, $S$ its unit sphere, and $f$ be as above. Then $(S,(\phi \circ f)(\|x+y\|))$ is a two-dimensional transitional probability space for every $\phi \in \Phi_{3}$.

It is clear that using the subclasses $\Phi_{2}$ and $\Phi_{3}$, we can obtain important special cases of the above theorem.

Our next corollary answers a question due to Mielnik ${ }^{1}$ concerning necessary and sufficient conditions for the existence of a particular type of twodimensional probability space in the setting of normed real linear spaces.

Corollary 2. Let $N$ be a normed real linear space, $S$ its unit sphere, and let $p(x, y)=\phi\left(\|x+y\|^{2} / 4\right)$ where $\phi \in \Phi_{0}$. Then $(S, p)$ is a two-dimensional transitional probability space if and only if $N$ is an inner product space and $\phi \in \Phi_{3}$.

The proof follows from the observation that if $f \in \mathcal{F}$ and $\phi \in \Phi_{3}$, then $\phi \circ f \in \mathscr{F}$, and the fact that $f(t)=(t / 2)^{2}$ is in $\mathscr{F}$.

Corollary 2 allows us to construct fairly bizarre two-dimensional transitional probability structures even in a real inner product space. Although $f(\|x+y\|)$ and, in particular, $\|x+y\|^{2} / 4$, are very well-behaved functions of $\|x+y\|$, one can take $\phi \in \Phi_{3}$ to be a Cantor singular function or any other solution of the functional equation $\phi(t)+\phi(1-t)=1$, constructed by the method of Ganguli [3]. This shows that if the model $(S, p)$ is not adequate for the polarization states $S$, one can construct more suitable models $(S, \phi \circ p)$ due to the variety of functions in $\Phi_{3}$, or even in $\Phi_{1}$. In the case where we have socalled "partial" or "incomplete" polarization, the representation space for reasonable transition probabilities must be a uniformly convex normed real linear space as is shown in [4].

\footnotetext{
1 In a private communication to the second author.
} 


\section{REFERENCES}

1. C. V. Stanojević, Mielnik probability spaces and characterization of inner product spaces, Trans. Amer. Math. Soc. 183 (1973), 441-448. MR 48 \#6904.

2. B. Mielnik, Geometry of quantum states, Comm. Math. Phys. 9 (1968), 55-80. MR 37 \#156.

3. P. L. Ganguli, A note on the integral of some classes of functions, Bull. Calcutta Math. Soc. 57 (1965), 63-68. MR 35 \# 1733.

4. A. R. Blass and C. V. Stanojević, Partial Mielnik spaces and characterization of uniformly convex spaces, Proc. Amer. Math. Soc. 55 (1976), 75-82.

Mathematics Department, University of Missouri-Rolla, Rolla, Missouri 65401 